\title{
Research
}

\section{Open-access transvaginal sonography in women of reproductive age with abnormal vaginal bleeding:}

\author{
a descriptive study in general practice
}

\begin{abstract}
Background

Diagnostic ultrasonography is used by GPs in approximately $10 \%$ of patients of reproductive age with abnormal vaginal bleeding. Transvaginal sonography is recommended as a first-line diagnostic instrument for assessing uterine pathology.

\section{Aim}

To assess if findings resulting from openaccess sonography were in agreement with the GPs' working hypotheses and if these findings contributed to GPs' management.

\section{Design and setting}

Prospective observational cohort study of GPs working in the health district of the Academic Medical Center, Amsterdam and their patients consulting with abnormal vaginal bleeding.
\end{abstract}

\section{Method}

Data on patients' history, GPs' primary working hypotheses, and intended management were recorded. After sonography, GPs recorded their actual management.

\section{Results}

A total of 122 patients were included by 18 GPs from June 2003 to December 2004. Data from 89 patients were available for analysis. The GPs working hypotheses implied 'no structural pathology' in 65/89 patients, and 'fibroids' in $24 / 89$ patients. Sonographic findings were confirmed in 50/65 patients where no structural pathology', and in 14/24 of those where 'fibroids' were expected. Initially, GPs had intended to refer nine patients to a gynaecologist. Actual management after sonographic assessment was watchful waiting or drug therapy in 57/89 patients. Eighty-nine per cent of these patients had normal sonographic findings. The actual referral rate rose to 27/89 patients. In 17 referred patients, sonographic findings were suggestive of intracavitary abnormalities.

\section{Conclusion}

Open-access sonography contributed to more accurate diagnoses and improved GPs management of women with abnormal vaginal bleeding

\section{Keywords}

primary health care; ultrasonography; uterine hemorrhage.

\section{INTRODUCTION}

For women of reproductive age, a change in menstrual bleeding pattern or interference of bleeding with daily life is frequently a reason for consulting their GP.1-4 Hormonal disturbances are thought to be the main cause of this abnormal vaginal bleeding Other causes include uterine fibroids, endometrial polyps, clotting disorders, cervical abnormalities, and chlamydia infection. Bleeding may also be related to complications of early pregnancy. Rarely, abnormal bleeding may be caused by gynaecological cancer in women of reproductive age. ${ }^{5-7}$

GPs often use descriptive diagnoses such as heavy menstrual bleeding, irregular bleeding, or intermenstrual bleeding. ${ }^{4,5}$ They use diagnostic ultrasonographic procedures in approximately $10 \%$ of patients with abnormal bleeding. ${ }^{8.9}$ If abnormal bleeding seems to be caused by a structural uterine abnormality, primary care guidelines for initial management of abnormal uterine bleeding recommend the use of transvaginal sonography as a firstline diagnostic instrument. 5.10

Offering GPs open-access sonography may assist them in making a more precise diagnosis and could result in a change in primary care management. However, the clinical value of sonography for abnormal bleeding in general practice has not yet

CJH de Vries, MD, GP; M Wieringa-de Waard, MD, $\mathrm{PhD}$, professor of general practice, Division of Clinical Methods and Public Health, Department of General Practice; WM Ankum, MD, PhD, gynaecologist, Department of Obstetrics and Gynaecology, Academic Medical Center, University of Amsterdam, The Netherlands. PJE Bindels, MD, $\mathrm{PhD}$, professor of general practice, Department of General Practice, Erasmus University Medical Centre, Rotterdam, The Netherlands.

Address for correspondence

Corlien JH de Vries, Division of Clinical Methods been evaluated, either in terms of establishing a more precise working hypothesis, or in terms of its impact on further management for individual patients. To provide more insight into the potential role of sonography in the management of women with abnormal vaginal bleeding in primary care, a prospective observational study was conducted.

\section{METHOD}

\section{Setting}

The study was carried out among 18 GPs providing care for 27000 patients, including 8025 women of reproductive age laged 20-55 years), from June 2003 to December 2004. Except for one GP from an adjacent rural area, all participating GPs worked in the urban health district of the Academic Medical Center in Amsterdam. Patients of reproductive age who presented with abnormal vaginal bleeding were eligible for inclusion. The study did not use predefined criteria for abnormal bleeding in terms of the bleeding pattern and its duration, heaviness, or frequency, but followed the subjective perception of the patient. 5 Women were excluded if they were less than 20 years of age, were pregnant, had started using hormonal contraceptives, or had had an intrauterine contraceptive device (IUCD) inserted within 3 months before consultation. Also, women with

and Public Health, Department of General Practice, Academic Medical Center, University of Amsterdam, Meibergdreef 15, 1105 AZ Amsterdam, The Netherlands.

E-mail: corlien.devriesdamc.uva.nl

Submitted: 15 November 2009; Editor's response: 1 June 2010; final acceptance: 31 August 2010. (c)British Journal of General Practice This is the full-length article (published online 31 May 2011) of an abridged version published in print. Cite this article as: Br J Gen Pract 2011; DOI: 10.3399/bjgp11X578016. 


\section{How this fits in}

Open-access transvaginal sonographic assessment revealed disagreement between GPs' working hypotheses and actual sonographic findings. Some of the problems detected by sonography, for example displaced IUCDs, could easily be solved by GPs, while a large proportion of patients with normal scans were able to be reassured with much more confidence than would have been the case if sonography had not been used. Overall, in a relatively high proportion of patients (23/89), anomalies amenable to hysteroscopic treatment were revealed.

postmenopausal bleeding, and those with a previous hysterectomy, were excluded.

\section{Data collection}

After informed consent had been obtained, GPs filled in a standardised questionnaire. The questionnaire addressed the patient's history lbleeding pattern, duration of symptoms, hormonal contraceptive use), findings at physical examination, and the GP's working hypothesis and intended management lpredefined as watchful waiting', start or stop of medication, additional investigations to be performed, referral to a gynaecologist, or any other management).

GPs were instructed to choose only one of the following predefined working hypotheses in the questionnaire: abnormal bleeding caused by hormonal contraceptive use loral contraceptive pill or progestogen use), by chlamydia infection, by fibroids, by an IUCD, or without underlying cause; that is, dysfunctional bleeding, or resulting from other causes.

\section{Transvaginal sonography}

All included patients underwent standardised transvaginal sonography at the outpatient clinic of the Department of Obstetrics and Gynaecology at the Academic Medical Centre, Amsterdam. Sonography was performed by experienced gynaecologists using a $5-7 \mathrm{MHz}$ transvaginal probe. A cervical chlamydiapolymerase chain reaction (PCR) test was performed immediately before every sonography. The sonographic findings were categorised as (1) normal findings, (2) fibroids, or (3) other findings. Normal findings were defined as a uterus with a regular contour, a homogenously structured myometrium, or small $1<3 \mathrm{~cm}$ in size) fibroids, and a straight regular endometrial line and ovaries without cysts or other abnormalities. ${ }^{11}$ Small fibroids were assessed as 'normal findings'. Current opinion is that the presence of these small fibroids has no clinical consequences. ${ }^{10,12}$ Predefined cut-off values for endometrial thickness were not used, since proper criteria are lacking. ${ }^{13}$

Fibroids $\geq 3 \mathrm{~cm}$ in size, or a uterus with multiple fibroids were categorised as fibroids. Sonographic findings suggestive of intracavitary abnormalities were also assessed as fibroids because of the clinical consequences - namely the necessity to carry out additional investigations. ${ }^{10,12}$ Coincidental abnormal structural findings such as a sonographic lucent ovarian cyst $>3 \mathrm{~cm}$ in size were clustered as other findings'. A displaced IUCD was defined as being in the cervical position or partial cervical position. No validation of sonographic findings was carried out, as the interobserver agreement of experienced gynaecologists is good. ${ }^{14}$

\section{Classification of working hypotheses and sonographic findings}

To explore the value of sonography, the working hypotheses were related to the sonographic findings. The working hypotheses were therefore clustered into (1) 'no structural pathology', and (2) 'fibroids'. The working hypotheses 'bleeding due to hormonal contraceptive use', 'bleeding as a result of chlamydia infection', 'bleeding caused by an IUCD', or 'bleeding without underlying cause' were assessed as primary working hypotheses implying no structural pathology.

The working hypothesis no structural pathology' was considered to be in agreement with normal sonographic findings. The working hypothesis 'fibroids' was classified as being in agreement with sonographic fibroids $\geq 3 \mathrm{~cm}$ in size, multiple fibroids, and/or intracavitary abnormalities. Although GPs were not able to detect intracavitary abnormalities without sonography, these abnormalities were arbitrarily categorised as 'fibroids' because of their clinical relevance for carrying out additional investigations.

\section{Contribution of sonography to patient management}

To assess the contribution of sonography to the actual management of women with abnormal bleeding. GPs filled in a second questionnaire once sonography had been performed and its findings had been discussed with the patient. The second questionnaire addressed the GP's diagnosis 


\begin{tabular}{|c|c|}
\hline \multicolumn{2}{|l|}{ Characteristic } \\
\hline Number of patients & 89 \\
\hline Median age (IQR), years & $+2136-46$ \\
\hline \multicolumn{2}{|l|}{ Bleeding pattern, $n$} \\
\hline Heavy & 25 \\
\hline Combination & 25 \\
\hline Intermenstrual & 22 \\
\hline Irregular & 17 \\
\hline \multicolumn{2}{|l|}{ Duration of symptoms, $n$} \\
\hline$<3$ months & 33 \\
\hline$\geq 3$ months & 56 \\
\hline \multicolumn{2}{|l|}{ Hormonal contraceptive use, $n$} \\
\hline None or sterilisation & 57 \\
\hline Oral contraceptive pill & 14 \\
\hline IUCD & 14 \\
\hline Copper & 10 \\
\hline Levonorgestrel-releasing & 4 \\
\hline Progestogen (continuous) & 4 \\
\hline \multicolumn{2}{|l|}{ Country of origin, $n$} \\
\hline Netherlands & 49 \\
\hline Surinam or Netherlands Antilles & 27 \\
\hline Other ${ }^{\mathrm{a}}$ & 13 \\
\hline \multicolumn{2}{|c|}{ 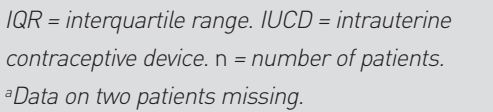 } \\
\hline
\end{tabular}

in view of sonographic findings, and the actual management that had been implemented - 'watchful waiting', start or stop medication, referral to a gynaecologist, or other type of management.

\section{Analysis}

Patient characteristics lage, bleeding pattern, duration of symptoms, contraceptive use, country of origin) were expressed using descriptive statistics. GPs' working hypotheses were expressed in numbers. In order to explore the value of sonography, analysis was carried out to assess whether sonographic findings had confirmed the primary working hypotheses 'no structural pathology' and 'fibroids', or if they had resulted in other findings. GPs were instructed to choose only one working hypothesis. If, despite this request, GPs chose multiple working hypotheses without ranking them, these cases were excluded.

The impact of sonographic findings on GPs' management was expressed in numbers. The initial referral rate and the actual management were described according to sonographic findings. Data were analysed in SPSS for Windows Iversion 17.0)

\section{RESULTS}

\section{General characteristics}

The participating GPs enrolled 122 patients (range: 1-20 patients per GP). Ten patients were lost to follow-up because they did not go back to their GP after sonography. In another seven patients, data on the working hypothesis or management were missing. Data of a further 16 patients were excluded, as the GPs filled in multiple working hypotheses without ranking. This resulted in a total of 89 patients available for analysis (Table 1). The median age of the patients was 42 years (interquartile range 36-46 years). Both heavy bleeding and a combination of bleeding patterns were the most frequently reported symptoms for 25 patients. Almost two-thirds (56/89) had had symptoms for $\geq 3$ months. Additionally, 57/89 did not use hormonal contraceptives or had been sterilised. Fifty-five per cent (49/85) of the patients were of Dutch origin.

\section{Working hypotheses}

GPs expected no structural pathology in 65 patients. The working hypothesis no underlying cause' was most frequently chosen in 37/65 patients, and other hypotheses implying no structural pathology in 28/65 patients. Fibroids were assumed in 24 patients.

With one exception, all included patients were screened for cervical chlamydia by means of a PCR test. In three patients, chlamydia infection was suspected, but PCR tests were negative. In two other patients, the PCR test was unexpectedly positive. Both patients had normal sonographic findings, suggesting that chlamydia infection was the sole cause of abnormal bleeding.

\section{Sonographic findings}

Sonography showed normal findings in 60/89 patients (Table 2). Sonographic findings suggestive of intracavitary abnormalities were the most frequently encountered abnormal findings: in 16 patients as a single finding, and in seven in combination with fibroids $\geq 3 \mathrm{~cm}$ in size, or multiple fibroids. Fibroids $\geq 3 \mathrm{~cm}$ in size or multiple fibroids ranked second, in 10 patients. A displaced copper IUCD was seen in five patients, that is, half of the copper IUCD users (5/10) (data not shown). Furthermore, sonography revealed an ovarian cyst in four patients - in two as a single finding, and in the other two in combination with other findings. In one patient, sonography showed a dehiscent cesarean section scar.

\section{Agreement between working hypotheses} and sonographic findings

In 50/65 patients with a working hypothesis 'no structural abnormality', indeed no 


\section{Table 2. Agreement between sonographic findings and GPs' working hypotheses $(n=89)$}

\begin{tabular}{|c|c|c|c|}
\hline \multirow[b]{2}{*}{ Sonographic findings } & \multicolumn{3}{|c|}{ Working hypotheses, $n$} \\
\hline & lo structural pathology & Fibroids & Total \\
\hline Normal findings & 50 & 10 & 60 \\
\hline \multicolumn{4}{|l|}{ Fibroids } \\
\hline Suggestive of intracavitary abnormality & 10 & 6 & 16 \\
\hline Fibroids $\geq 3 \mathrm{~cm}^{\mathrm{a}}$ & 2 & 8 & 10 \\
\hline Other findings ${ }^{b}$ & 3 & 0 & 3 \\
\hline Total & 65 & 24 & 89 \\
\hline
\end{tabular}

abnormalities were detected by sonography (Table 2). Unexpected fibroids were present in 2/65 of these patients. Moreover, sonographic findings suggestive of intracavitary abnormalities were present in $10 / 65$ of patients in whom no structural abnormalities were expected.

Fibroids suspected by the GP were confirmed in 14/24 patients. In 8/24 of these patients, fibroids $\geq 3 \mathrm{~cm}$ in size or multiple fibroids were revealed, and in 6/24 patients there were findings suggestive of intracavitary abnormalities. In 10/24 patients, sonography yielded normal findings.

\section{Contribution of sonographic findings to management}

The GPs' actual management after sonographic assessment was watchfu waiting or drug therapy in 57/89 patients (Table 3). The majority of these patients (51/57) had normal sonographic findings. Watchful waiting or drug therapy was also the GPs actual management in 5/23 patients with sonographic findings suggestive of intracavitary abnormalities. The initially intended referral rate rose from 9/89 to 27/89 after sonography. The majority of the referred patients (17/27) had sonographic findings suggestive of intracavitary abnormalities.

\section{DISCUSSION}

\section{Summary}

Open access to transvaginal sonography offered a relevant contribution to the diagnostic and therapeutic management of women with abnormal vaginal bleeding in primary care. Among the GPs variety of working hypotheses expressed as possible causes of abnormal bleeding, the majority implied 'no structural pathology'. The agreement between normal sonographic findings and GP's working hypotheses 'no structural pathology' was 50/65. The agreement between sonographic fibroids and the working hypothesis 'fibroids' was 14/24. In 23/89 patients, sonography yielded findings suggestive of intracavitary abnormalities. Sonographic findings allowed GPs to carry out actual management, mainly in terms of wellfounded watchful waiting or drug therapy in $57 / 89$ patients, and an increased referral rate for $27 / 89$ patients. In a relatively high proportion of patients (23/89), anomalies amenable to hysteroscopic treatment were revealed. In addition, some problems revealed by ultrasonography could be dealt with in primary care, for example, displaced IUCD.

\section{Strength and limitations}

For the first time, this study allows an insight into both the underlying pathology and the contribution of sonography to management of women of reproductive age who consult their GPs for abnormal vaginal bleeding. Some limitations of the study need to be addressed here.

First, the question arises as to whether the patients included are representative of those encountered in primary care. The possibility of selection bias in this study, induced by participating GPs who may have included only those patients in whom underlying pathology was suspected, cannot be excluded entirely. However, the patients included in the study seem to reflect a typical general practice population, as only $9 / 89$ of them were initially considered for referral to a gynaecologist. The included patients showed a variety of bleeding patterns, which were very similar to those found in another study performed in general practice by Shapley et al. ${ }^{4}$ This confirms that the study population reflects normal general practice. Further confirmation of this is provided by Emanuel et al, who found a normal uterine cavity in $63 \%$ of patients referred for abnormal uterine bleeding. ${ }^{15}$ In the present study, a normal uterine cavity was found in $74 \%$ 
Table 3. Sonographic findings and actual management after sonography $(n=89)$

\begin{tabular}{|c|c|c|c|c|c|}
\hline \multirow[b]{2}{*}{ Sonographic findings } & \multicolumn{3}{|c|}{ Actual management, $n$} & \multirow[b]{2}{*}{ Additional investigation } & \multirow[b]{2}{*}{ Total } \\
\hline & Watchful waiting & Medication $^{a}$ & Referral & & \\
\hline Normal findings & 33 & 18 & 5 & 4 & 60 \\
\hline \multicolumn{6}{|l|}{ Fibroids } \\
\hline Suggestive of intracavitary abnormality & 1 & 2 & 13 & 0 & 16 \\
\hline Fibroids $\geq 3 \mathrm{~cm}^{b}$ & 0 & $3^{c}$ & $6^{d}$ & $1 e$ & 10 \\
\hline Other findings ${ }^{f}$ & 0 & 0 & 3 & 0 & 3 \\
\hline Total & 34 & 23 & 27 & 5 & 89 \\
\hline
\end{tabular}

(66/89) of patients, a sufficiently higher prevalence than was found in referred patients. In the Netherlands, the proportion of women aged $18-45$ years taking oral contraception is much higher $(41 \%]^{16}$ than the $16 \%(14 / 89)$ seen in the present study population. This may be explained by the fact that the use of oral contraceptives reduces and probably even prevents the occurrence of heavy and irregular bleeding, thus reducing the probability of its users being entered into the study. ${ }^{17}$ Compared with the general Dutch population, the present study population contained more patients of non-Western ethnicity. These were mainly black women from Surinam and the Netherlands Antilles, a population well known for its high prevalence of fibroids. ${ }^{18}$

Secondly, in the Netherlands, gynaecological ultrasound scans are usually done by gynaecologists, as in the present study, rather than by ultrasonographers. Therefore, some effect of inflating the actual referral number cannot be completely ruled out. The participating gynaecologists filled in a standardised sonography form and were instructed to refer the patient back to their GP for further management. In practice, gynaecologists advocate additional diagnostic work-up and removal of intracavitary abnormalities such as submucous fibroids and endometrial polyps..$^{10,19}$ It might have been difficult for the GP not to refer. On the other hand, all patients contacted their GP for management after the ultrasound scan, and $5 / 23$ patients with intracavitary abnormalities were not referred. The researchers did not ask the GPs to explain the motivation behind their management. Reasons for first-line treatment instead of referral might be mild symptoms, the patient's preference, or GP-related factors and preferences. $^{20}$ Since the study was performed, the actual advocated management on abnormal bleeding has not been changed in more recent guidelines in the Netherlands. ${ }^{21}$

Third, despite vaginal sonography being generally accepted as a useful first-line diagnostic procedure, its accuracy varies depending on the type of uterine abnormality. In diagnosing intracavitary abnormalities in particular, transvaginal sonography is not considered as being the gold standard, but is generally accepted as being the first diagnostic step. ${ }^{10,12,13}$ Obviously, the diagnostic accuracy of vaginal sonography was beyond the scope of the present study.

Apart from the impact of sonographic findings on GPs' management of patients, some other important observations were made. A remarkably high number of displaced copper IUCDs was found - in 5/10 patients who had one. Displaced IUCDs are quite common, and apart from causing abnormal bleeding, are thought to be less effective in preventing pregnancies. A recent study reported that displaced copper IUCDs occurred in $29 \%$ of patients during an observation period up to 60 months. ${ }^{22}$ GPs should be aware of partial IUCD expulsion as a cause of abnormal vaginal bleeding, a complication that is easily detected by ultrasonography.

\section{Comparison with existing literature}

Some guidelines on abnormal vaginal bleeding recommend initial symptombased medical treatment without further investigations. 5.10 However, more information about normal sonographic findings may confirm the GP's diagnosis and may imply a solid argument for prescribing medical therapy to wellinformed patients in general practice. ${ }^{20,23-25}$ Sonography is a safe procedure that is well- 
tolerated by patients. ${ }^{26}$ Nonetheless, vaginal sonography is an intimate procedure that might cause distress and discomfort for some women. Therefore, adequate information about the procedure is required. ${ }^{27,28}$

To date, little is known about the costeffectiveness of diagnostic procedures in patients with abnormal uterine bleeding. ${ }^{29}$ Julian et al carried out a prospective nonrandomised comparative study of a GP-led integrated care pathway and a consultantled one-stop menstrual clinic for referred patients with menorrhagia. ${ }^{30}$ After 8 months, there was no difference in treatment between the two groups, except for fewer outpatient appointments in the GP-led care pathway. In the present study, open-access sonography resulted in an increased number of referred patients who, outside this study, would have stayed under primary care. Obviously, some of these women would probably have been referred anyway, albeit at a later stage. The consequences of this change in management in terms of cost-effectiveness deserve further evaluation.

Giving GPs open access to transvaginal sonography revealed the disagreement between their working hypotheses and sonographic findings. The contribution of this study to the management of women with normal scans is that GPs were able to reassure their patients with more confidence. It was also beneficial to those women with findings amenable to gynaecological treatment, who, because of the additional information from sonography, were referred more efficiently and without undue delay. This is especially important for those women with intracavitary abnormalities, amenable for hysteroscopic resection, which cannot be suspected and detected by any other means. In addition, some problems revealed by sonography could be solved in primary care, for example displaced IUCDs.

\section{Implications for research and practice}

Future research should focus on the yield of transvaginal sonography in primary care patients with abnormal vaginal bleeding in relation to symptom reduction and GP management, the patients' perspectives, and cost-effectiveness. 


\section{REFERENCES}

1. Linden van der MW, Westert GP, Bakker de DH, Schellevis FG. Tweede nationale studie naar ziekten en verrichtingen in de huisartspraktijk. Klachten en aandoeningen in de bevolking en in de huisartspraktijk. [Health complaints and diseases in the population and in general practice]. Second Dutch National Survey of General Practice.] Utrecht: NIVEL, 2004

2. Royal College of General Practitioners, Office of Population Censuses and Surveys, and Department of Health. Morbidity statistics from general practice. Fourth national study 1991-1992. London: HMSO, 1995.

3. Shapley M, Jordan K, Croft PR. Increased vaginal bleeding: the reasons women give for consulting primary care. J Obstet Gynaecol 2003; 23(1): 48-50.

4. Shapley M, Jordan K, Croft PR. Abnormal bleeding patterns associated with menorrhagia in women in the community and in women presenting to primary care. Fam Pract 2007; 24(6): 532-537.

5. Meijer LJ, Zwart SJ, Westerveld MC, et al. NHG-standaard Vaginaal bloedverlies, eerste herziening. [Dutch College of General Practitioners. Revised practice guideline, vaginal bleeding.] Huisarts Wet 2001; 44: 260-266.

6. Goodman A. Terminology and evaluation of abnormal uterine bleeding in premenopausal women. http://www.uptodate.com/contents/terminology-andevaluation-of-abnormal-uterine-bleeding-in-premenopausal-women laccessed 28 Apr 2011).

7. Shapley M, Jordan J, Croft PR. A systematic review of postcoital bleeding and risk of cervical cancer. Br J Gen Pract 2006; 56(527): 453-460.

8. Grant C, Gallier L, Fahey T, et al. Management of menorrhagia in primary care-impact on referral and hysterectomy: data from the Somerset Morbidity Project. J Epidemiol Community Health 2000; 54(9): 709-713.

9. De Vries CJ, Wieringa-de Waard M, Vervoort CL, et al. Abnormal vaginal bleeding in women of reproductive age: a descriptive study of initial management in general practice. BMC Women's Health 2008; 8: 7.

10. National Institute for Health and Clinical Excellence. Heavy menstrual bleeding. Clinical guideline 44. London: National Institute for Health and Clinical Excellence, 2007.

11. Nederlandse Vereniging voor Obstetrie en Gynaecologie (NVOG). Richtlijn 27 Gynaecologische echoscopie. [Dutch Society of Obstetrics and Gynaecology. Practice guideline 27. Gynaecological ultrasonography]. Utrecht: NVOG, 1997.

12. Nederlandse Vereniging voor Obstetrie en Gynaecologie (NVOG). Richtlijn menorrhagie. (Dutch Society of Obstetrics and Gynaecology. Practice guideline Menorrhagia]. Utrecht: NVOG, 2003

13. Farquhar C, Ekeroma A, Furness S, Arroll B. A systematic review of transvaginal ultrasonography, sonohysterography and hysteroscopy for the investigation of abnormal uterine bleeding in premenopausal women. Acta Obstet Gynecol Scand 2003; 82(6): 493-504.

14. Emanuel MH, Ankum WH, Verdel MJC, Hart AAM. The reproducibility of transvaginal sonography of the uterus in patients with abnormal uterine bleeding. Ultrasound Obstet Gynecol 1996; 8(5): 346-349.

15. Emanuel MH, Verdel MJC, Stas H, et al. An audit of true prevalence of intrauterine pathology: the hysteroscopical findings controlled for patien selection in 1202 patients with abnormal uterine bleeding. Gyn Endoscopy
1995; 4: 237-241.

16. Centraal Bureau voor de Statistiek. Geboorteregeling; anticonceptiemethode en leeftijd van de vrouw 2003. [Office for National Statistics. Birth control: contraceptive method and women's age 2003.

http://statline.cbs.nl/StatWeb/publication/?DM=SLNL\&PA=37459\&D1=028\&D2=0\&D3=2\&WW=T (accessed 28 Mar 2011).

17. Farquhar C, Brown J. Oral contraceptive pill for heavy menstrual bleeding Cochrane Database of Syst Rev 2009; (2): CD000154.

18. Marshall LM, Spiegelman D, Barbieri RL, et al. Variation in incidence of uterine leiomyoma among premenopausal women by age and race. Obstet Gynecol 1997; 90(6): 967-973.

19. Timmermans A, van Dongen $\mathrm{H}$, Mol BW, et al. Hysteroscopy and removal of endometrial polyps: a Dutch survey. Eur J Obstet Gynecol Reprod Biol 2008 138(1): 76-79.

20. O'Flynn N, Britten N. Diagnosing menstrual disorders: a qualitative study of the approach of primary care professionals. Br J Gen Pract 2004; 54(502): 353-358.

21. Meijer LJ, Bruinsma ACA, Drost B, et al. NHG-standaard Vaginaal bloedverlies, tweede herziening. [Dutch College of General Practitioners. Revised practice guideline, vaginal bleeding.] Huisarts Wet 2008; 3: 128-137.

22. Merki-Feld GS, Schwarz D, Imthurn B, Keller PJ. Partial and complete expulsion of the Multiload 375 IUD and the levonorgestrel-releasing IUD after correct insertion. Eur J Obstet Gynecol Reprod Biol 2008; 137(1): 92-96.

23. Santer M, Wyke S, Warner P. What aspects of periods are most bothersome for women reporting heavy menstrual bleeding? Community survey and qualitative study. BMC Women's Health 2007; 7: 8 .

24. O'Flynn N. Menstrual symptoms: the importance of social factors in women's experiences. Br J Gen Pract 2006; 56(533): 950-957.

25. Protheroe J, Chew-Graham C. The role of primary care in diagnosis and management of menorrhagia; a qualitative study of women with menorrhagia. Prim Health Care Research and Development 2005; 6: 217-223.

26. Critchley HOD, Warner $\mathrm{P}$, Lee A, et al. Evaluation of abnormal uterine bleeding comparison of three outpatient procedures within cohorts defined by age and menopausal status. Health Technol Assess 2004; 8(34): iii-iv, 1-139.

27. Cowan Bennett C, Richards DS. Patient acceptance of endovaginal ultrasound. Ultrasound Obstet Gynecol 2000; 15(1): 52-55.

28. Loken K, Steine S, Laerum E. Patient satisfaction and quality of care at four diagnostic imaging procedures: mammography, double-contrast barium enema, abdominal ultrasonography and vaginal ultrasonography. Eur Radiol 1999; 9(7): 1459-1463

29. Clark J. Cost-effectiveness of diagnostic strategies for the management of abnormal uterine bleeding (heavy menstrual bleeding and post-menopausal bleeding): systematic reviews, IPD meta-analysis and model based economic evaluation. Southampton: NIHR Health technology Assessment Programme, 2010. http://www.hta.ac.uk/project/2145.asp laccessed 28 Mar 2011).

30. Julian S, Naftalin NJ, Clark M, et al. An integrated care pathway for menorrhagia across the primary-secondary interface: patients' experience, clinical outcomes, and service utilisation. Qual Saf Health Care 2007: 16(2): 110-115. 